\title{
Diverse microRNAs with convergent functions regulate tumorigenesis (Review)
}

\author{
MIN-YAN ZHU ${ }^{1 *}$, WEI ZHANG ${ }^{2 *}$ and TAO YANG ${ }^{1}$ \\ ${ }^{1}$ Research Center for Translational Medicine, Shanghai East Hospital, Tongji University School of Medicine, \\ Shanghai 200120; ${ }^{2}$ Department of Pharmacology, School of Basic Medicine, Hebei University of Chinese Medicine, \\ Shijiazhuang, Hebei 050200, P.R. China
}

Received November 26, 2014; Accepted November 16, 2015

DOI: $10.3892 / \mathrm{ol} .2015 .4020$

\begin{abstract}
MicroRNAs (miRNAs) regulate several biological processes, including tumorigenesis. In order to comprehend the roles of miRNAs in cancer, various screens were performed to investigate the changes in the expression levels of miRNAs that occur in different types of cancer. The present review focuses on the results of five recent screens, whereby a number of overlapping miRNAs were identified to be downregulated or differentially regulated, whereas no miRNAs were observed to be frequently upregulated. Furthermore, the majority of the miRNAs that were common to $>1$ screen were involved in signaling networks, including wingless-related integration site, receptor tyrosine kinase and transforming grow th factor- $\beta$, or in cell cycle checkpoint control. The present review will discuss the aforementioned miRNAs implicated in cell cycle checkpoint control and signaling networks.
\end{abstract}

\section{Contents}

1. Introduction

2. Commonly downregulated microRNAs

3. Differentially regulated microRNAs in various types of cancer

4. Signalling cascades with microRNA interaction

5. Conclusion

Correspondence to: Professor Tao Yang, Research Center for Translational Medicine, Shanghai East Hospital, Tongji University School of Medicine, 150 Jimo Road, Shanghai 200120, P.R. China E-mail: mryangtao2005@yahoo.com

\section{*Contributed equally}

Key words: microRNA, cancer stem cell, tumorigenesis, signal transduction, microarray

\section{Introduction}

MicroRNAs (miRNAs) are small non-coding RNAs of 21-25 nucleotides in length that control gene expression via post-transcriptional regulation (1). Contrasting with the maturation process of normal coding RNAs, the miRNA genes are initially transcribed by RNA polymerase II into a primary transcript in the nucleus, where the hairpin structure is processed into precursor miRNA by a microprocessing complex that includes Drosha and DiGeorge syndrome chromosomal (or critical) region 8 (DGCR8) (1). Subsequently, the $\sim 70$ nucleotide-long precursor miRNA is exported into the cytoplasm, where it undergoes secondary processing by Dicer, and one strand of the hairpin is then incorporated into a ribonucleoprotein complex known as miRNA-induced silencing complex (1). Once matured, a single miRNA may target miscellaneous messenger RNAs (mRNAs), and an individual mRNA may be regulated by various miRNAs (1).

miRNAs have significant roles during stem cell maintenance and differentiation (2). In addition, they have frequently been used as markers for certain types of cancer cells, by comparing the measured levels of known miRNAs with those present in their wild-type counterparts $(2,3)$. Screens investigating differential expression of miRNAs in various cancer cell populations have been previously performed. The present review will focus on the results of five screens conducted in the past recent years, which investigated prostate cancer (3), neuroblastoma (4), pancreatic cancer (5), chronic myeloid leukemia (CML) (6) and osteosarcoma cells (7). Specifically, Liu et al (3) used three prostate cancer metastatic cell lines, namely LAPC9, LAPC4 and Du145 [bone cluster of differentiation (CD) $44^{+} / \mathrm{CD}^{4} 4^{-}$subpopulation sorting based on LAPC9, LAPC4 and Du145 cell lines; side population (SP)/non-SP sorting based on LAPC9 cell line; and CD133 ${ }^{+} / \mathrm{CD} 133^{-}$subpopulation sorting based on LAPC4 cell line], and compared the expression levels of miscellaneous miRNAs (with vs. without markers) in these cells. Following testing for 324 DGCR8 miRNAs by reverse transcription-quantitative polymerase chain reaction, 137 miRNAs were identified to be expressed in the three cell types, of which, 4 were downregulated, confirming that miRNAs may be differentially expressed in various cancer cell populations (3). Samaraweera et al (4) compared the miRNA expression levels of two types of tumorigenic 
neuroblastic cells, namely stem cells (I-cells) and neuronal cells (N-cells), with those of non-tumorigenic non-neuronal cells (S-cells) by microarray analysis. A total of 313 distinct miRNAs were assayed, among which, 17 displayed reduced expression levels and 3 displayed increased expression levels in neuronal cells, compared with non-neuronal cells. A total of 11 miRNAs were observed to be downregulated in I-cells, compared with N-cells (4). Consistently, the differential expression profile of miRNAs in distinct cell compartments applies also to pancreatic cancer, CML and osteosarcoma, as demonstrated by the following studies: Bao et al (5) isolated triple positive $\mathrm{CD} 44^{+} / \mathrm{CD} 133^{+} /$epithelial cell adhesion molecule ${ }^{+}$cells from two pancreatic cancer cell lines termed MiaPaCa-2 and L3.6pl, and identified >400 miRNAs that were differentially expressed in these cancer stem cell-like populations by microarray analysis. Using this type of analysis, Zhu et al (6) studied the differences in expression levels of 275 known human miRNAs in bone marrow stromal cells derived from patients with CML and normal human subjects, and identified 19 downregulated and 6 upregulated miRNAs. Furthermore, Feng et al (7) compared the gene expression profile of the osteosarcoma stem cell line 3AB-OS with that of its parental cell line MG63 by microarray analysis, and identified 189 differentially expressed miRNAs in addition to chromosome copy number variations and dysregulated genes.

In the present review, those miRNAs that were identified to be differentially expressed in $\geq 2$ of the aforementioned screens are discussed, and their interaction with signaling networks is emphasized. A notable point is that, although certain miRNAs were observed to be commonly downregulated or differentially regulated in the above screens, no miRNA was identified to be commonly upregulated in those studies (Table I).

\section{Commonly downregulated microRNAs}

Let-7. The let-7 family of miRNAs was initially identified in Caenorhabditis elegans, where it participates in the transition of later larval to adult stage (8). The let-7 family has 13 members in humans, namely let-7a-1, a-2, a-3, 7b, 7c, $7 \mathrm{~d}, 7 \mathrm{e}, 7 \mathrm{f}-1,7 \mathrm{f}-2,7 \mathrm{~g}, 7 \mathrm{i}$, miR-98 and miR-202 (9,10). In the aforementioned screens, the let-7 family members involved were all identified to be downregulated. Thus, in prostate cancer and osteosarcoma, 4 members of the let-7 family were downregulated (let-7a, b, e and f), whereas in pancreatic cancer and CML let-7a and let-7f were observed to be downregulated (3,5-7). Let-7 is highly conserved across bilaterians, and is involved in developmental switches in C. elegans and mammals (11). It has been reported that let-7 may be involved in neural cell proliferation and differentiation in C. elegans, Drosophila and mammals (12-14). In addition to its developmental roles, let-7 has also been identified to be involved in neuronal cell aging by suppressing the expression of abnormal cell lineage protein 41 (LIN-41). In old neurons, let-7 binds to the 3 ' untranslated region of the $L I N-41$ gene and inhibits its expression, which results in the downregulation of the axonal regeneration abilities of the aged anterior ventral microtubule axon in C. elegans (15). In cancer cells, the majority of the let-7 family members were observed to be downregulated (16). It has been reported that let-7 manifests tumor suppressive effects in prostate cancer cells (17). Furthermore, differential mechanisms between let-7 and miR-34a on the regulation of the cell cycle in prostate cancer cells have been previously reported. Thus, miR-34a primarily induced G1 phase cell cycle arrest followed by cell senescence, while let-7 mainly induced G2/M arrest (17).

miR-34 $a$ and miR-183. miR-34a and miR-183 were identified to be downregulated in $\mathrm{CD} 44^{+}$prostate cancer and osteosarcoma cells $(3,7)$. Enforced expression of miR-34a in bulk or purified CD $44^{+}$prostate cancer cells was demonstrated to inhibit clonogenic expansion, tumor regeneration and metastasis (3). In addition, CD44 has been identified as a direct and functional target of miR-34a, which may induce G1 phase cell cycle arrest (17). The miR-34 family members may be induced by tumor protein p53, and may be able to directly suppress epithelial-mesenchymal transition (EMT) by inhibiting the expression of Snail, a transcription factor required for EMT (18). In addition, miR-34 has been observed to associate the tumor suppressor p53 to the wingless-related integration site (Wnt) signaling pathway by directly binding to the 3' untranslated region of wingless-type mouse mammary tumor virus integration site family, member 1 (WNT1), WNT3, low density lipoprotein receptor-related protein 6 (LRP6), axis inhibition protein 2 (AXIN2), $\beta$-catenin and lymphoid enhancer-binding factor 1 (LEF1) (19). Therefore, the expression of miR-34 may be capable of inhibiting metastasis (20). The miR-183 family of genes, including miR-96, -182 and -183, are expressed coordinately in the sensory cells of zebrafish, chicken and mouse (21-26), where they are required for sensory cell differentiation and functioning. Ueno et al (27) observed that miR-183 was able to suppress the expression of Dickkopf-related protein 3 and mothers against decapentaplegic homolog 4 (Smad4) in prostate cancer, resulting in tumor growth inhibition. Additionally, it has been demonstrated that miR-183 is able to suppress the metastasis of osteosarcoma cells via downregulation of the expression of Ezrin (28).

miR-15. miR-15 was identified to be downregulated in screens of $\mathrm{CD}_{133^{+}}$subpopulation and SP of prostate cancer and osteosarcoma cells $(3,7)$. The $m i R-15$ gene is located on the chromosome $13 \mathrm{q} 14$, and was previously identified to be downregulated in chronic lymphocytic leukemia and pituitary adenomas (29,30). miR-15 downregulates oncogenes, including B-cell lymphoma 2, myeloid cell leukemia 1, cyclin D1 and WNT3A (31). The repression of miR-15 and miR-16 in hypoxia was identified to be caused by hypoxia inducible factor-2 $\alpha$ via c-myc signaling (32).

miR-130a. miR-130a was observed to be downregulated in pancreatic cancer and $\operatorname{CML}(5,6)$. miR-130a is a c-myc-responsive gene, which has been identified to regulate CCAAT/enhancer-binding protein $\beta$, homeobox A5, runt-related transcription factor 3 and Wnt signaling (33-35). Previous studies have demonstrated that the expression of miR-130a is increased in non-small cell lung cancer (36), whereas in glioblastoma patients, increased levels of miR-130a are associated with long-term survival (37). 
Table I. Summary of miRs reviewed in the present study.

\begin{tabular}{lccc}
\hline miR & Cancer screen where miR was identified & Signaling pathway involved & Expression levels \\
\hline let-7 & Prostate cancer/osteosarcoma & Development and LIN-41 & Downregulated \\
miR-34a & Prostate cancer/osteosarcoma & Snail, p53 and Wnt & Downregulated \\
miR-183 & Prostate cancer/osteosarcoma & DKK3 and Smad4 & Downregulated \\
miR-15 & Prostate cancer/osteosarcoma & BCL2, MCL1, CCND1 and WNT3A & Downregulated \\
miR-130a & Pancreatic cancer/chronic myeloid leukemia & C/EBP, HOXA5, RUNX3 and Wnt & Downregulated \\
miR-21 & Pancreatic cancer cells/neuroblastoma & PTEN and SPRY1 & Differentially regulated \\
miR-335 & Pancreatic cancer cells/neuroblastoma & TGF, RTK, ATM and Oct4-pRB & Differentially regulated \\
miR-133a & Prostate cancer/osteosarcoma & Snail and others & Differentially regulated
\end{tabular}

miR, microRNA; LIN-41, abnormal cell lineage protein 41; p53, tumor protein P53; Wnt, wingless-related integration site; DKK3, Dickkopf-related protein 3; Smad4, mothers against decapentaplegic homolog 4; BCL2, B-cell lymphoma 2; MCL1, myeloid cell leukemia 1; CCND1, cyclin D1; WNT3A, wingless-type mouse mammary tumor virus integration site family, member 3A; C/EBP, CCAAT/enhancer-binding protein $\beta$; HOXA5, homeobox A5; RUNX3, runt-related transcription factor 3; PTEN, phosphatase and tensin homolog; SPRY1, sprouty homolog 1; TGF, transforming growth factor; RTK, receptor tyrosine kinase; ATM, ataxia telangiectasia mutated; Oct4, octamer-binding transcription factor 4; $\mathrm{pRB}$, retinoblastoma protein.

\section{Differentially regulated microRNAs in various types of cancer}

miR-21. In $\mathrm{CD}_{133^{+}}$prostate cancer cells, miR-21 was identified to be upregulated, whereas in neuroblastoma it was observed to be downregulated $(3,4)$. In pancreatic cancer, miR-21-3p was observed to be upregulated, whereas miR-21-5p was observed to be downregulated (5). The $m i R-21$ gene is located on the chromosome 17q23.2, and its potential targets include phosphatase and tensin homolog (PTEN) and sprouty homolog 1 (SPRY1) (38). The expression levels of miR-21 have been revealed to correlate with cell proliferation in hepatocellular cancer (39). It has additionally been reported that miR-21 is able to downregulate the tumor suppressor programmed cell death 4, and therefore promote the metastatic activities of colorectal and gastric cancer $(40,41)$.

miR-335. miR-335 is upregulated in pancreatic cancer, but downregulated in tumorigenic neuroblastoma cells $(5,7)$. Lynch et al (42) reported that miR-335 was able to suppress metastasis of neuroblastoma cells by directly repressing the downstream effector proteins of the transforming growth factor (TGF)- $\beta$ pathway, namely Rho-associated, coiled-coil containing protein kinase 1 (ROCK1) and mitogen-activated protein kinase 1 (MAPK1), which consequently reduced the phosphorylation levels of myosin light chain and inhibited the invasiveness of neuroblastoma cells. In addition to its regulatory function in signaling pathways, miR-335 has roles in at least two pathways involved in cell cycle control, namely ataxia telangiectasia mutated (ATM)-dependent DNA damage control (43) and octamer-binding transcription factor 4-retinoblastoma protein (Oct4-pRB)-dependent stem cell renewal/cell cycle control (44). In the ATM pathway, irradiation-activated ATM downregulates miR-335 via cyclic adenosine monophosphate response element binding protein, which subsequently activates $\mathrm{C}$ terminal binding protein interaction protein, which in turn recruits breast cancer 1 , early onset to double strand breaks (43). During stem cell renewal, miR-335 is able to regulate the cell cycle by controlling the activities of the Oct4-pRb signaling pathway. When differentiation begins, miR-335 is upregulated, and thus downregulates the expression of Oct4, which impairs the rapid division cycle of stem cell renewal (44). Previous studies have demonstrated that the expression levels of miR-335 are cancer type-specific. Thus, downregulation of miR-335 is observed in certain types of metastatic breast cancer (45), whereas upregulation is observed in the breast cancer cell line MCF7 (43). Regarding its role in ATM-dependent DNA damage control, Martin et al (43) suggested that differential expression levels of miR-335 may explain the variations in terms of resistance to chemotherapy or radiotherapy observed among different patients.

miR-133a. miR-133a was identified to be downregulated in $\mathrm{CD}_{133}{ }^{+}$prostate cancer cells but upregulated in osteosarcoma cells $(3,7)$. The location and expression of miR-133a, as well as its target genes, have been previously reviewed elsewhere (46), and therefore will not be discussed in the present review. A notable point is that in the majority of the cancer stem cells listed in the present review, miR-133a was observed to be downregulated (46). Numerous overexpression studies have previously demonstrated that miR-133a is able to effectively suppress tumor growth $(17,46)$. Accordingly, the observation that miR-133a was upregulated in osteosarcoma was unexpected (7). miR-133a was also observed to be expressed in cultured embryonic rat cardiomyocytes (7). Furthermore, a recent study demonstrated that miR-133a was able to promote cardiac reprogramming via suppression of the Snaill gene (47). Based on the differences between osteosarcoma and prostate or other types of cancer such as lung cancer, it is possible to conclude that, since osteosarcoma is a mesodermal tissue-derived tumor, this may explain the upregulation of miR-133a observed in 3AB-OS osteosarcoma cells $(7,17)$. Nevertheless, additional studies are required to validate this hypothesis. 


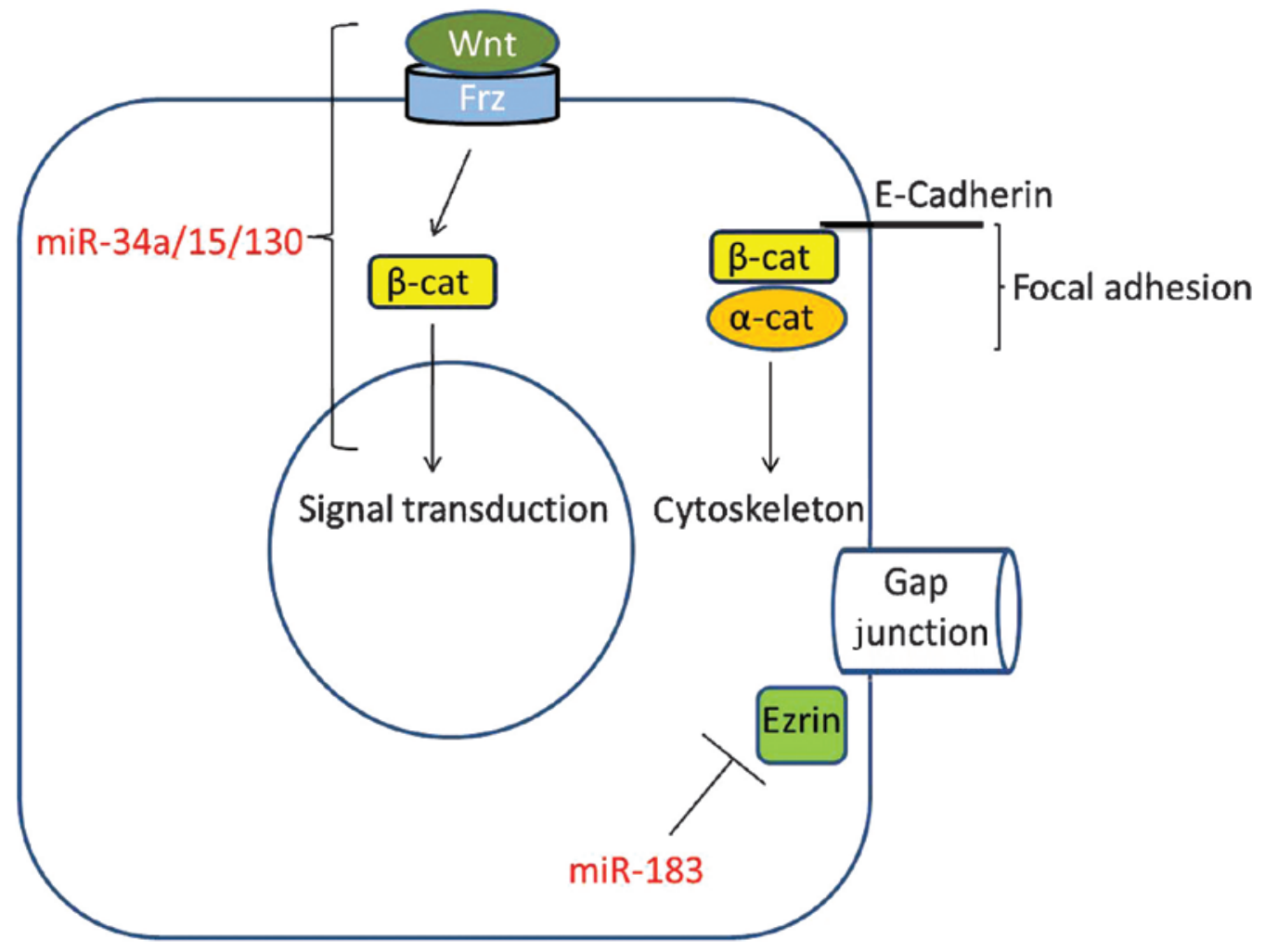

Figure 1. Wnt signaling interacts with intercellular junction components. $\beta$-catenin is involved in Wnt signaling and focal adhesions. $\beta$-catenin is a key downstream target of the Wnt signaling pathway and is also an important part of intercellular junctions. The loss of cell-cell contact in the epithelium leads to the formation of cancer cells, which is the initial stage of the metastatic process. Miscellaneous miRs from previous screens discussed in the present review were identified to be involved in this complicated network. Wnt, wingless-related integration site; miR, microRNA; $\beta$-cat, $\beta$-catenin; $\alpha$-cat, $\alpha$-catenin; Frz, frizzy.

\section{Signalling cascades with microRNA interaction}

Prior to miRNAs becoming a relevant topic in cancer research, signaling networks were considered the key regulators of tumor development, and this vision currently remains (1). The miRNAs listed in the preceding sections of the present review have been previously associated with Wnt, receptor tyrosine kinase (RTK) and TGF- $\beta /$ bone morphogenetic protein (BMP) signaling pathways, which involve genes encoding cell cycle checkpoint proteins and transcriptional regulatory proteins $(1,20,48)$.

Wnt. Wnt signaling is dysregulated in various types of cancer, as discussed elsewhere (48). The crosstalk between miR-30 family members and the canonical Wnt signaling pathway has been discussed in detail previously (49). Previous studies have implicated miR-34a, miR-15 and miR-130 in the regulation of Wnt signaling, and these miRNAs, in addition to miR-183, were observed to be downregulated in prostate cancer and osteosarcoma cells $(3,5,7)$. Wht signaling is relevant in terms of one of its key downstream components, namely $\beta$-catenin. This protein is a signaling molecule and a component of focal adhesions, which forms a complex with E-cadherin and $\alpha$-catenin (50). Therefore, miR-183 may be involved in the regulatory network of intercellular junctions (Fig. 1), since miR-183 downregulates the expression of Ezrin (a key component of the gap junction of adjacent epithelial cells), thus potentially inhibiting the invasion and metastasis of osteosarcoma cells (28).
$T G F-\beta / B M P$. BMP signaling is a type of TGF- $\beta$ signaling that is considered to be the 'master regulator' of EMT and cancer metastasis (51). Accordingly, it is not surprising that miRNA-335, which functions to repress ROCK1 (the downstream target of TGF- $\beta$ ), is downregulated in tumorigenic neuroblastoma cells (42). In addition, miR-335 represses MAPK1, which is a common downstream factor in RTK signaling, while miR-183 represses the expression of Smad4, thus inhibiting BMP signaling (52). Therefore, miRNAs appear to directly regulate the expression of key signaling molecules and provide an additional regulatory layer for the signaling network.

RTK. SPRY1 and PTEN (targeted by miR-21), as well as MAPK1 (targeted by miR-335), are downstream factors of RTK signaling, which includes signal transduction induced by epidermal growth factor, fibroblast growth factor, vascular endothelial growth factor and insulin-like growth factor signals (53). All these signaling pathways have been implicated in cancer initiation and development $(53,54)$. miR-21 regulates RAS1, a key signaling molecule that is a common downstream target of RTK signals, which is located downstream of SPRY1 and PTEN and upstream of MAPK1, and participates in the growth of diverse types of cancer (39-41).

Cell cycle checkpoints. It has been previously reported that miR-34 may be induced by p53 and subsequently cause G1 phase cell cycle arrest $(3,17)$, while miR-335 may be downregulated by activated ATM (43). Therefore, it appears that the genes involved in cell cycle checkpoint control are primarily 
upstream of miRNA expression. The loss of checkpoint control is required for cancer initiation and development $(43,44)$. Thus, there is a clear direct connection between miRNAs and cancer formation, which will not be discussed further in the present review. As previously indicated, miR-34 may be capable of binding to the 3' untranslated region of WNT1, WNT3, LRP6, AXIN2, $\beta$-catenin and LEF1, thus suppressing the Wnt signaling pathway (19). These scenarios suggest that miRNAs possess a bridging role, connecting Wnt signaling with cell cycle control. Future studies may confirm additional miRNAs to act by bridging the gap between signal transduction processes and cell cycle control.

\section{Conclusion}

The majority of miRNAs observed to be downregulated during tumor progression are considered to be tumor suppressive, and may be under the control of tumor suppressors such as p53. miRNAs regulate signal transduction by modulating the expression levels of key regulatory proteins, which may be involved in identical or differing signaling pathways to those of their corresponding miRNA. In addition, miRNAs may be modulated by cell cycle checkpoint proteins, thus connecting signaling networks with cell cycle control. Compared with previous studies on protein-encoding genes, the current research on miRNAs is at its initial stages, and it is predicted that the functions exhibited by different miRNAs may be as complex as those displayed by protein-encoding genes. In conclusion, the functional regulatory mechanisms of a biological entity are composed of protein-encoding and non-encoding genes, including miRNAs, which together fine-tune the processes that form a living organism.

\section{Acknowledgements}

The present study was financially supported by the National Natural Science Foundation of China (Beijing, China; grant no's. 81372750 and 81572518), the Shanghai Natural Science Foundation of China (Shanghai, China; grant no. 12ZR1425200), the Science \& Technology Development Fund of Shanghai Pudong (Shanghai, China; grant no. PKJ2014-Y10) and the Scientific Research Foundation for the Returned Overseas Chinese Scholars from the State Education Ministry of China (Beijing, China; grant no. 2013-1792).

\section{References}

1. Bartel DP: MicroRNAs: Genomics, biogenesis, mechanism, and function. Cell 116: 281-297, 2004.

2. Gangaraju VK and Lin H: MicroRNAs: Key regulators of stem cells. Nat Rev Mol Cell Biol 10: 116-125, 2009.

3. Liu C, Kelnar K, Liu B, Chen X, Calhoun-Davis T, Li H, Patrawala L, Yan H, Jeter C, Honorio S, et al: The microRNA miR-34a inhibits prostate cancer stem cells and metastasis by directly repressing CD44. Nat Med 17: 211-215, 2011.

4. Samaraweera L, Grandinetti KB, Huang R, Spengler BA and Ross RA: MicroRNAs define distinct human neuroblastoma cell phenotypes and regulate their differentiation and tumorigenicity. BMC Cancer 14: 309, 2014.

5. Bao B, Ali S, Ahmad A, Li Y, Banerjee S, Kong D, Aboukameel A, Mohammad R, Van Buren E, Azmi AS and Sarkar FH: Differentially expressed miRNAs in cancer-stem-like cells: Markers for tumor cell aggressiveness of pancreatic cancer. Stem Cells Dev 23: 1947-1958, 2014.
6. Zhu X, Lin Z, Du J, Zhou X, Yang L and Liu G: Studies on microRNAs that are correlated with the cancer stem cells in chronic myeloid leukemia. Mol Cell Biochem 390: 75-84, 2014.

7. Feng Z, Takahashi R, Nakamura T, Sato D, Shirasawa N, Nakayama A, Kurashige S, Kosawada T, Kitajima T and Umezu M: Expression of microRNA-1, microRNA-133a and Hand2 protein in cultured embryonic rat cardiomyocytes. In Vitro Cell Dev Biol Anim 50: 700-706, 2014.

8. Reinhart BJ, Slack FJ, Basson M, Pasquinelli AE, Bettinger JC Rougvie AE, Horvitz HR and Ruvkun G: The 21-nucleotide let-7 RNA regulates developmental timing in Caenorhabditis elegans. Nature 403: 901-906, 2000

9. Thornton JE and Gregory RI: How does Lin28 let-7 control development and disease? Trends Cell Biol 22: 474-482, 2012.

10. Ruby JG, Jan C, Player C, Axtell MJ, Lee W, Nusbaum C, Ge H and Bartel DP: Large-scale sequencing reveals 21U-RNAs and additional microRNAs and endogenous siRNAs in C. elegans. Cell 127: 1193-1207, 2006.

11. Ambros V: MicroRNAs and developmental timing. Curr Opin Genet Dev 21: 511-517, 2011.

12. Abrahante JE, Daul AL, Li M, Volk ML, Tennessen JM, Miller EA and Rougvie AE: The Caenorhabditis elegans hunchback-like gene lin-57/hbl-1 controls developmental time and is regulated by microRNAs. Dev Cell 4: 625-637, 2003.

13. Lin SY, Johnson SM, Abraham M, Vella MC, Pasquinelli A Gamberi C, Gottlieb E and Slack FJ: The $C$ elegans hunchback homolog, hbl-1, controls temporal patterning and is a probable microRNA target. Dev Cell 4: 639-650, 2003.

14. Zhao C, Sun G, Li S, Lang MF, Yang S, Li W and Shi Y: MicroRNA let-7b regulates neural stem cell proliferation and differentiation by targeting nuclear receptor TLX signaling. Proc Natl Acad Sci USA 107: 1876-1881, 2010.

15. Zou Y, Chiu H, Zinovyeva A, Ambros V, Chuang CF and Chang C: Developmental decline in neuronal regeneration by the progressive change of two intrinsic timers. Science 340: 372-376, 2013.

16. Boyerinas B, Park SM, Hau A, Murmann AE and Peter ME: The role of let-7 in cell differentiation and cancer. Endocr Relat Cancer 17: F19-F36, 2010.

17. Liu C, Kelnar K, Vlassov AV, Brown D, Wang J and Tang DG: Distinct microRNA expression profiles in prostate cancer stem/progenitor cells and tumor-suppressive functions of let-7. Cancer Res 72: 3393-3404, 2012.

18. Kim NH, Kim HS, Li XY, Lee I, Choi HS, Kang SE, Cha SY, Ryu JK, Yoon D, Fearon ER, et al: A p53/miRNA-34 axis regulates Snail1-dependent cancer cell epithelial-mesenchymal transition. J Cell Biol 195: 417-433, 2011.

19. Cha YH, Kim NH, Park C, Lee I, Kim HS and Yook JI: MiRNA-34 intrinsically links p53 tumor suppressor and Wnt signaling. Cell Cycle 11: 1273-1281, 2012.

20. Rokavec M, Öner MG, Li H, Jackstadt R, Jiang L, Lodygin D, Kaller M, Horst D, Ziegler PK, Schwitalla S, et al: IL-6R/STAT3/miR-34a feedback loop promotes EMT-mediated colorectal cancer invasion and metastasis. J Clin Invest 124: 1853-1867, 2014.

21. Wienholds E, Kloosterman WP, Miska E, Alvarez-Saavedra E, Berezikov E, de Bruijn E, Horvitz HR, Kauppinen S and Plasterk RH: MicroRNA expression in zebrafish embryonic development. Science 309: 310-311, 2005.

22. Shao P, Zhou H, Xiao ZD, He JH, Huang MB, Chen YQ and Qu LH: Identification of novel chicken microRNAs and analysis of their genomic organization. Gene 418: 34-40, 2008.

23. Kloosterman WP, Wienholds E, de Bruijn E, Kauppinen S and Plasterk RH: In situ detection of miRNAs in animal embryos using LNA-modified oligonucleotide probes. Nat Methods 3: 27-29, 2006.

24. Weston MD, Pierce ML, Rocha-Sanchez S, Beisel KW and Soukup GA: MicroRNA gene expression in the mouse inner ear. Brain Res 1111: 95-104, 2006.

25. Xu S, Witmer PD, Lumayag S, Kovacs B and Valle D: MicroRNA (miRNA) transcriptome of mouse retina and identification of a sensory organ-specific miRNA cluster. J Biol Chem 282: 25053-25066, 2007.

26. Weston MD, Pierce ML, Jensen-Smith HC, Fritzsch B, Rocha-Sanchez S, Beisel KW and Soukup GA: MicroRNA-183 family expression in hair cell development and requirement of microRNAs for hair cell maintenance and survival. Dev Dyn 240: 808-819, 2011.

27. Ueno K, Hirata H, Shahryari V, Deng G, Tanaka Y, Tabatabai ZL, Hinoda Y and Dahiya R: MicroRNA-183 is an oncogene targeting Dkk-3 and SMAD4 in prostate cancer. Br J Cancer 108: $1659-1667,2013$. 
28. Zhu J, Feng Y, Ke Z, Yang Z, Zhou J, Huang X and Wang L: Down-regulation of miR-183 promotes migration and invasion of osteosarcoma by targeting Ezrin. Am J Pathol 180: 2440-2451, 2012.

29. Calin GA, Dumitru CD, Shimizu M, Bichi R, Zupo S, Noch E, Aldler H, Rattan S, Keating M, Rai K, et al: Frequent deletions and down-regulation of micro- RNA genes miR15 and miR16 at 13q14 in chronic lymphocytic leukemia. Proc Natl Acad Sci USA 99: 15524-15529, 2002.

30. Bottoni A, Piccin D, Tagliati F, Luchin A, Zatelli MC and degli Uberti EC: miR-15a and miR-16-1 down-regulation in pituitary adenomas. J Cell Physiol 204: 280-285, 2005.

31. Aqeilan RI, Calin GA and Croce CM: miR-15a and miR-16-1 in cancer: Discovery, function and future perspectives. Cell Death Differ 17: 215-220, 2010.

32. Xue G, Yan HL, Zhang Y, Hao LQ, Zhu XT, Mei Q and Sun SH: c-Myc-mediated repression of miR-15-16 in hypoxia is induced by increased HIF-2 $\alpha$ and promotes tumor angiogenesis and metastasis by upregulating FGF2. Oncogene 34: 1393-1406, 2015.

33. Yang F, Miao L, Mei Y and Wu M: Retinoic acid-induced HOXA5 expression is co-regulated by HuR and miR-130a. Cell Signal 25: 1476-1485, 2013.

34. Xu N, Shen C, Luo Y,Xia L, Xue F, Xia Q and Zhang J: Upregulated miR-130a increases drug resistance by regulating RUNX3 and Wnt signaling in cisplatin-treated HCC cell. Biochem Biophys Res Commun 425: 468-472, 2012.

35. Larsen MT, Häger M, Glenthøj A, Asmar F, Clemmensen SN, Mora-Jensen H, Borregaard N and Cowland JB: miRNA-130a regulates $\mathrm{C} / \mathrm{EBP}-\varepsilon$ expression during granulopoiesis. Blood 123: 1079-1089, 2014

36. Wang XC, Tian LL, Wu HL, Jiang XY, Du LQ, Zhang H, Wang YY, Wu HY, Li DG, She Y, et al: Expression of miRNA-130a in nonsmall cell lung cancer. Am J Med Sci 340: 385-388, 2010.

37. Qiu S, Lin S, Hu D, Feng Y, Tan Y and Peng Y: Interactions of miR-323/miR-326/miR-329 and miR-130a/miR-155/miR-210 as prognostic indicators for clinical outcome of glioblastoma patients. J Transl Med 11: 10, 2013.

38. Haghikia A and Hilfiker-Kleiner D: MiRNA-21: A key to controlling the cardiac fibroblast compartment? Cardiovasc Res 82: $1-3,2009$.

39. Meng F, Henson R, Wehbe-Janek H, Ghoshal K, Jacob ST and Patel T: MicroRNA-21 regulates expression of the PTEN tumor suppressor gene in human hepatocellular cancer. Gastroenterology 133: 647-658, 2007.

40. Asangani IA, Rasheed SA, Nikolova DA, Leupold JH, Colburn NH, Post S and Allgayer H: MicroRNA-21 (miR-21) post-transcriptionally downregulates tumor suppressor Pdcd4 and stimulates invasion, intravasation and metastasis in colorectal cancer. Oncogene 27: 2128-2136, 2008.
41. Li L, Zhou L, Li Y, Lin S and Tomuleasa C: MicroRNA-21 stimulates gastric cancer growth and invasion by inhibiting the tumor suppressor effects of programmed cell death protein 4 and phosphatase and tensin homolog. J BUON 19: 228-236, 2014.

42. Lynch J, Fay J, Meehan M, Bryan K, Watters KM, Murphy DM and Stallings RL: MiRNA-335 suppresses neuroblastoma cell invasiveness by direct targeting of multiple genes from the non-canonical TGF- $\beta$ signalling pathway. Carcinogenesis 33: 976-985, 2012.

43. Martin NT, Nakamura K, Davies R, Nahas SA, Brown C, Tunuguntla R, Gatti RA and Hu H: ATM-dependent MiR-335 targets CtIP and modulates the DNA damage response. PLoS Genet 9: e1003505, 2013.

44. Schoeftner S, Scarola M, Comisso E, Schneider C and Benetti R: An Oct4-pRb axis, controlled by MiR-335, integrates stem cell self-renewal and cell cycle control. Stem Cells 31: 717-728, 2013.

45. Tavazoie SF, Alarcón C, Oskarsson T, Padua D, Wang Q, Bos PD, Gerald WL and Massagué J: Endogenous human microRNAs that suppress breast cancer metastasis. Nature 451: 147-152, 2008.

46. Nohata N, Hanazawa T, Enokida H and Seki N: MicroRNA-1/133a and microRNA-206/133b clusters: Dysregulation and functional roles in human cancers. Oncotarget 3: 9-21, 2012.

47. Muraoka N, Yamakawa H, Miyamoto K, Sadahiro T, Umei T, Isomi M, Nakashima $\mathrm{H}$, Akiyama M, Wada R, Inagawa $\mathrm{K}$, et al: MiR-133 promotes cardiac reprogramming by directly repressing Snail and silencing fibroblast signatures. EMBO J 33: 1565-1581, 2014.

48. Atlasi Y, Looijenga L and Fodde R: Cancer stem cells, pluripotency, and cellular heterogeneity: A WNTer perspective. Curr Top Dev Biol 107: 373-404, 2014.

49. Zhao JJ and Carrasco RD: Crosstalk between microRNA30a/b/c/d/e-5p and the canonical Wnt pathway: Implications for multiple myeloma therapy. Cancer Res 74: $5351-5358,2014$

50. Fagotto F: Looking beyond the Wnt pathway for the deep nature of $\beta$-catenin. EMBO Rep 14: 422-433, 2013.

51. Fazilaty H, Gardaneh M, Bahrami T, Salmaninejad A and Behnam B: Crosstalk between breast cancer stem cells and metastatic niche: Emerging molecular metastasis pathway? Tumour Biol 34: 2019-2030, 2013

52. Miyazono K: Signal transduction by bone morphogenetic protein receptors: Functional roles of Smad proteins. Bone 25: 91-93, 1999.

53. Shibuya M: VEGFR and type-V RTK activation and signaling. Cold Spring Harb Perspect Biol 5: a009092, 2013.

54. Wang D, Du L, Liu Q, Liu X and Wang Z: Receptor tyrosine kinase alterations and therapeutic opportunities in squamous cell carcinoma of the lung. Cancer Chemother Pharmacol 72: 725-731, 2013. 